\title{
Is Osteoarthritis of the Knee Hereditary? A Review of the Literature
}

\section{Clement ND*}

Speciality Trainee Trauma and Orthopaedics, United Kingdom

\begin{abstract}
Osteoarthritis (OA) of the knee joint is a common cause of disability. This review presents the current evidence and knowledge regarding the genetic inheritability of OA of the knee, and a contemporary hypothesis for progression of the disease. The current evidence would suggest there is a hereditary predisposition to OA of the knee. However, this would seem to be multifactorial and the development of OA in the knee may depend upon multiple genetic factors, not only of those relating to cartilage degeneration but also of those relating to the morphology of the knee joint, both of these factors seem to be influenced by similar genes. Knowledge of these genes that affect the initiation and/ or progression of knee OA are important for development of new treatment options at an early stage of the disease preventing future physical disability.
\end{abstract}

Keywords: Osteoarthritis; Genetic inheritability; Genetic factors

\section{Introduction}

Osteoarthritis (OA) of the knee joint is a common cause of disability, especially among the elderly population where it is the most prevalent co morbidity to result in functional disability [1]. Primary OA of the knee is, by definition an idiopathic disease that occurs without any known initiating factor. The macroscopic features that typify OA are: softening, splitting, and fibrillation of articular cartilage, in association with subchondral bone sclerosis, cysts, and osteophytes [2]. The prevalence of OA seems to be increasing; a recent study from the United States demonstrated a $29 \%$ increase over a 10 year period, affecting to 27 million people in 2005 [3]. Furthermore, a recent the Framingham Osteoarthritis Study demonstrated a two to three fold increase in the rate of symptomatic OA of the knee over the last 20 years [4]. The hereditary nature of Heberden's nodes, osteophytes of the distal interphalangeal joints of the hands, was described in the nineteenth century [5] and by the middle of the last century this phenotype was demonstrated to have a dominant inherited trait [6]. This type of OA is termed nodal OA, which often affects multiple sites and may have a polygenic inheritance [7]. However, OA may be also termed local, being confined to a single joint such as the knee.

Knowledge of the genetic influence contributing to OA of the knee has important clinical repercussions. Potentially if the genes associated with OA of knee were known this would give us a better understanding of the molecular pathogenesis, which may enable therapeutic intervention before the osteoarthritis results in disability. Currently the only treatment of end stage OA of the knee is a total knee replacement (TKR), which is an effective procedure and approximately 64,000 are performed each year in the United Kingdom [8]. However, only four out of five patients undergoing TKR are satisfied with their outcome, with the remainder being unsure or dissatisfied [9]. In addition there are other health risks associated to TKR, which is associated with morbidity and mortality [10]. Hence, the current treatment of end stage OA of the knee is not optimal, and if we could prevent the occurrence and/ or progression of knee OA with knowledge of the genetic inheritability of OA this would have fantastic repercussions upon society relieving a substantial disability burden.

This review presents the current evidence and knowledge regarding the genetic inheritability of OA of the knee, and a contemporary hypothesis for progression of knee OA. The articles included in this review were identified from Pub Med and Medline searches using the key words: osteoarthritis, knee, and genetics, hereditary.

\section{Familial Risk of Developing OA of the Knee}

It is possible to calculate the risk ratio for a relative of an individual with $\mathrm{OA}$ of their knee, compared with the population prevalence. This risk ratio is a measure for familial aggregation [11], and has been applied extensively in the field of genetic epidemiology to detect a genetic linkage with a define pathology [12]. Hence, it is possible to identify subjects who have clinically severe disease, such as end stage $\mathrm{OA}$ of the knee that warrants TKR and to compare the rate (prevalence) of knee OA in their genetically related siblings, and assess their risk of OA compared to that of a matched population. This sib recurrence risk is termed the lambda sib $(\lambda S)$.

A study in Oxford compared the prevalence of TKR in siblings of individuals that had underwent TKR for end stage OA of the knee with the prevalence of TKR in a control group, which was defined as the spouse of that individual [13]. They demonstrated, using a group of 121 patients who had a TKR for end stage OA of the knee (probands), that their siblings were at a greater risk of needing a TKR relative to their spouse (Table 1). Neame et al. [14] conducted one of the largest genetic studies to date to determine whether there was a genetic contribution to knee OA which was defined by structural change on plain radiographs. They compared the prevalence of knee OA in the community with prevalence of knee OA among siblings of index cases with previously diagnosed knee OA. Using 490 knee OA index cases listed for TKR and 737 of their siblings, who were aged more than 40 years old, and 1729 controls, they demonstrate that siblings were at an increased risk of knee OA (Table 1). This difference remained after adjustment for important environmental risk factors. Hence, they concluded that siblings are at increased risk of knee OA in comparison with the general population. More recently McDonnell et al. [15] studied of 112 patients with primary anteromedial osteoarthritis of the knee and their families. Sibling risk was determined using randomly selected single siblings, and their spouses were used as controls to compare the rate of OA in their siblings. The presence of symptomatic osteoarthritis was determined using an Oxford knee score of 29 or more (12 being least symptomatic and 60 being the most symptomatic) and supported by a Kellgren and Lawrence radiological score of two or more. They found that there was

*Corresponding author: Clement ND, Speciality Trainee Trauma and Orthopaedics, Royal Infirmary of Edinburgh, Little France, EH16 4SA United Kingdom, Tel: 44-131-242-3497; Fax: 44-131-242-3541; E-mail: nickclement@doctors.org.uk

Received March 22, 2013; Accepted April 22, 2013; Published April 25, 2013

Citation: Clement ND (2013) Is Osteoarthritis of the Knee Hereditary? A Review of the Literature. Hereditary Genetics S1: 004. doi:10.4172/2161-1041.S1-004

Copyright: () 2013 Clement ND. This is an open-access article distributed under the terms of the Creative Commons Attribution License, which permits unrestricted use, distribution, and reproduction in any medium, provided the original author and source are credited. 
Citation: Clement ND (2013) Is Osteoarthritis of the Knee Hereditary? A Review of the Literature. Hereditary Genetics S1: 004. doi:10.4172/21611041.S1-004

Page 2 of 3

\begin{tabular}{|c|c|c|}
\hline Author (year) & Condition & Sib marker \\
\hline Chitnavis et al. (1997) [13] & TKR for OA & Sibs with TKR \\
\hline Neame et al. (2004) [14] & Tibiofemoral (TF) OA & Sibs with TKR \\
\hline Neame et al. (2004) [14] & Patellofemoral (PF) OA & Sibs with TKR \\
\hline Neame et al. (2004) [14] & Knee OA (TF or PF) & Sibs with TKR \\
\hline $\begin{array}{c}\text { McDonnell et al. [15] } \\
(2007)\end{array}$ & Anteromedial knee OA & Sibs with UKR \\
\hline
\end{tabular}

UKR, unicompartmental knee replacement

Table 1: Studies reporting the hereditary risk of OA of the knee for siblings compared to matched population controls.

a significant $(\mathrm{p}=0.03)$ increased risk of anteromedial OA relative to the control group (Table 1). This specific phenotype of anteromedial OA, with preservation of cartilage of other compartments of the knee, may be due to abnormal and/or increased loading and thus could be attributed to mechanical causes rather than genetic factors. However, the significant risk identified by this group for their cohort, and in conjunction with the other two studies reported, supports the fact that inherited genetic factors probably play a major role in the development of OA of the knee even for this particular phenotypic pattern of knee OA. All three studies seem to demonstrate that OA of the knee has a strong hereditary predisposition (Table 1).

Kellgren and Moore [16], more than 60 years ago, demonstrated for families that had an individual with generalised nodal OA, that involving six or more joint groups, were at a twofold excess of OA among first-degree relatives compared with population controls. This risk increased further for relatives if the index family member was of female gender. It is interesting to note that these recurrence risks are comparable to those $\mathrm{OA}$ of the knee illustrated in the table 1. However, a recent study in the Netherlands evaluated whether familial aggregation of OA differs by joint site in a sibling pair study of patients with OA at multiple sites [17]. The diagnosis of knee, hip, and spine OA was based on a combination of pain or stiffness on most days of the previous month and osteophytes or joint space narrowing identified on radiographic analysis. Hand osteoarthritis was defined by American College of Rheumatology criteria. There were 191 sibling pairs included, with a mean age of 60 years and $85 \%$ were women. In the index case (proband) OA was present in spine (76\%), hands (77\%), knees $(37 \%)$, and hips (26\%). The most common combinations in probands were spine-hand (59\%), spine-knee (27\%), and hand-knee (25\%). The risk was adjusted for age, sex, and body mass index for siblings to be affected in the same joint sites as the proband were increased for OA of the hand (odds ratio $=4.4,95 \%$ confidence interval 2.0 to 9.5), hip (odds ratio $=3.9,95 \%$ confidence interval 1.8 to 8.4 ), spine (odds ratio $=2.2$, $95 \%$ confidence interval 1.0 to 5.1 ), hip-spine (odds ratio $=4.7,95 \%$ confidence interval 2.1 to 10.4 ), and hand-hip (odds ratio=3.4, 95\% confidence interval 1.1 to 10.4). Siblings of probands with osteoarthritis in the knee did not have an increased likelihood of knee OA. Hence, they conclude in middle aged patients with familial osteoarthritis at multiple sites, familial aggregation of OA was most striking for hand and hip but remarkably absent for the knee. The reason why the knee was different from the other synovial joints assessed is not clear, but may relate to alterations in the joint architecture in association with a genetic predisposition that results in knee OA, as supported by those studies in the table 1 .

\section{Gene Mutations and Abnormal Joint Architecture Causing OA}

Sandell [18] in a recent review described the aetiology of osteoarthritis as a continuum, of defective genes that result in different disorders affecting the joint, that then lead to the development of
OA. A large proportion of the susceptibility to OA is thought to be due to inherited genetic factors, of which some are also associated in the developmental processes of the knee joint [18]. In the knowledge of this she proposed an alternative hypothesis for the origin of OA: "subtle (and not-so-subtle) alterations in the joint architecture determine whether the joint will be susceptible to degeneration". Gross developmental changes in joint morphology result in early-onset OA, which is associated with chondrodysplasia [19]. However, more subtle changes in joint architecture, that are not radio graphically evident, may predispose an individual to the development of OA later in life. In addition the genes that are associated with the developmental process of the knee joint are also associated with OA [18]. An understanding in the molecular pathways that these genes are involved in and the function of the specific proteins they encode, may provide insight into how these genes affect the developmental process and susceptibility to knee OA [20].

Sandell's [18] hypothesis requires a re-evaluation of what we consider to be primary and secondary OA of the knee. Primary OA of the knee is currently defined as that which there is no predisposing factors or structural abnormalities. In contrast to this, secondary OA of the knee is defined as that which results from a traumatic injury, specific anatomical deformities in the joint or specific abnormalities of the cartilage extracellular matrix. However, more recent novel analytical techniques using MRI analysis of the knee have provided more detailed structural information than traditional radiographic measurement of joint space narrowing [21]. Reichenbach et al. [21] revealed new information regarding subtle abnormalities of the joint architecture that are also present in patients with primary OA of the knee. Progress continues to be made regarding the early diagnosis of $\mathrm{OA}$ of the knee using biochemical biomarkers [22], which facilitates the detection of molecular events before potential tissue changes occur. This will hopefully increase our understanding and ability to treat $\mathrm{OA}$ of knee in the future. Together with these technical advances, the discovery of further genetic influences on the development of knee OA will improve our understanding of the pathogenesis of this disease, and the classification as Sandell suggests of primary and secondary OA may begin to blur.

\section{The Future Burden of Knee OA}

During the next decade it is predicted that within Western society there will be a marked increase in the elderly population, and currently the fastest growing population is the "oldest old" ( $>85$ years) [23]. The Scottish population over the age of 75 years is projected to increase by $81 \%$ by the year 2031 [24], and is one of the reasons why the rate of TKR has been steadily increasing during the last two decades [8]. This increasing burden upon the future society of patients with knee OA will have huge financial repercussions [25], and for some patients the risks associated with TKR may be too great and decline such surgery and live with their disability. Even just the thought of being able to understand the molecular pathways involved in development and progression of knee OA that may lead to treatments targeting specific pathways to 
prevent $\mathrm{OA}$ and the associated disability is an exciting prospect for the future.

\section{Future Research into the Hereditability of Knee OA}

To expand Sandell's [18] hypothesis further, there may be no such thing as primary OA of the knee and OA may only occur secondary to another cause, which may be known or unknown. This may be due to a mechanical alignment problem, abnormal joint architecture, or abnormal cartilage construct/repair mechanisms, hence the search for a single genetic link is futile because of the potentially multiple causes. Before any researcher carries outcome genetic studies to assess their association with the development of knee OA, it is import that a potential cause of their OA is identified, as to group them into a primary OA group would make such an association difficult to prove. Only now do we understand the influence of multiple secondary causes of hip OA. Current evidence would suggest there are multiple phenotypes that eventually lead to degenerative hip OA [26]. In addition during the last decade, with the use of modern magnetic resonance scanning, the concept of femoro-acetabula impingement has now been acknowledged as a secondary cause of hip OA which was previously thought to be of a primary cause [27]. This change in knowledge and understanding may result in specific genes being identified for each of these recently recognised secondary phenotypes/causes, and may actually identify those genes that cause primary OA of the hip, should it exist. To apply this same strategy to the understanding of knee OA may also result in more secondary causes being identified, for example with modern imaging techniques to identify abnormal joint architecture such as magnetic resonance scanning, that may then result in specific genes being identified for doffing pathologies of knee OA.

\section{Conclusion}

Current evidence would suggest there is a hereditary predisposition to $\mathrm{OA}$ of the knee. However, this would seem to be multifactorial and the development of $\mathrm{OA}$ in the knee may depend upon multiple genetic factors, not only of those relating to cartilage degeneration but also of those relating to the morphology of the knee joint, both of which seem to be influenced by similar genes. These genes that affect the joint structure may be expressed in cartilage, meniscus, tendons, fat pads or any other joint tissue, and consequently may be involved in the development of OA of the knee. Knowledge of the genes that affect the initiation and/or progression of knee OA are important for development of new treatment options at an early stage of the disease preventing future physical disability.

\section{References}

1. Guccione AA, Felson DT, Anderson JJ, Anthony JM, Zhang Y, et al. (1994) The effects of specific medical conditions on the functional limitations of elders in the Framingham Study. Am J Public Health 84: 351-358.

2. Dieppe P, Kirwan J (1994) The localization of osteoarthritis. Br J Rheumato 33: 201-203.

3. Lawrence RC, Felson DT, Helmick CG, Arnold LM, Choi H, et al. (2008) Estimates of the prevalence of arthritis and other rheumatic conditions in the United States. Part II. Arthritis Rheum 58: 26-35.

4. Nguyen US, Zhang Y, Zhu Y, Niu J, Zhang B, et al. (2011) Increasing prevalence of knee pain and symptomatic knee osteoarthritis: survey and cohort data. Ann Intern Med 155: 725-732.

5. Doherty M (2004) How important are genetic factors in osteoarthritis? J Rheumatol Suppl 70: 22-27.

6. Stecher RM, Hersh AH (1944) Heberden's nodes: the mechanism of inheritance in hypertrophic arthritis of the fingers. J Clin Invest 23: 699-704.

7. KELLGREN JH, LAWRENCE JS, BIER F (1963) GENETIC FACTORS IN GENERALIZED OSTEO-ARTHROSIS. Ann Rheum Dis 22: 237-255.
8. Culliford DJ, Maskell J, Beard DJ, Murray DW, Price AJ, et al. (2010) Tempora trends in hip and knee replacement in the United Kingdom: 1991 to 2006. Bone Joint Surg Br 92: 130-135.

9. Scott CE, Howie CR, MacDonald D, Biant LC (2010) Predicting dissatisfaction following total knee replacement: a prospective study of 1217 patients. J Bone Joint Surg Br 92: 1253-1258.

10. Clement ND, Jenkins PJ, Brenkel IJ, Walmsley P (2012) Predictors of mortality after total knee replacement: a ten-year survivorship analysis. J Bone Join Surg Br 94: 200-204.

11. Risch N (1990) Linkage strategies for genetically complex traits. I. Multilocus models. Am J Hum Genet 46: 222-228.

12. Guo SW (2002) Sibling recurrence risk ratio as a measure of genetic effect: caveat emptor! Am J Hum Genet 70: 818-819.

13. Chitnavis J, Sinsheimer JS, Clipsham K, Loughlin J, Sykes B, et al. (1997) Genetic influences in end-stage osteoarthritis. Sibling risks of hip and knee replacement for idiopathic osteoarthritis. J Bone Joint Surg Br 79:660-664

14. Neame RL, Muir K, Doherty S, Doherty M (2004) Genetic risk of knee osteoarthritis: a sibling study. Ann Rheum Dis 63: 1022-1027.

15. McDonnell SM, Sinsheimer J, Price AJ, Carr AJ (2007) Genetic influences in the aetiology of anteromedial osteoarthritis of the knee. J Bone Joint Surg Br 89: 901-903.

16. KELLGREN JH, MOORE R (1952) Generalized osteoarthritis and Heberden's nodes. Br Med J 1: 181-187.

17. Riyazi N, Meulenbelt I, Kroon HM, Ronday KH, Hellio le Graverand MP, et al. (2005) Evidence for familial aggregation of hand, hip, and spine but not knee osteoarthritis in siblings with multiple joint involvement: the GARP study. Ann Rheum Dis 64: 438-443.

18. Sandell LJ (2012) Etiology of osteoarthritis: genetics and synovial joint development. Nat Rev Rheumatol 8: 77-89.

19. Goldring MB, Goldring SR (2007) Osteoarthritis. J Cell Physiol 213: 626-634

20. Bos SD, Slagboom PE, Meulenbelt I (2008) New insights into osteoarthritis: early developmental features of an ageing-related disease. Curr Opin Rheumatol 20: 553-559.

21. Reichenbach S, Guermazi A, Niu J, Neogi T, Hunter DJ, et al. (2008) Prevalence of bone attrition on knee radiographs and MRI in a community-based cohort. Osteoarthritis Cartilage 16: 1005-1010.

22. van Spil WE, DeGroot J, Lems WF, Oostveen JC, Lafeber FP (2010) Serum and urinary biochemical markers for knee and hip-osteoarthritis: a systematic review applying the consensus BIPED criteria. Osteoarthritis Cartilage 18: 605612.

23. Office for National Statistics (2010). UK

24. General Register Office for Scotland (2010) UK.

25. Weinstein AM, Rome BN, Reichmann WM, Collins JE, Burbine SA, et al. (2013) Estimating the burden of total knee replacement in the United States. J Bone Joint Surg Am 95: 385-392.

26. Hamilton HW, Jamieson J (2012) The classification of degenerative hip disease. J Bone Joint Surg Br 94: 1193-1201.

27. Banerjee P, McLean CR (2011) Femoroacetabular impingement: a review of diagnosis and management. Curr Rev Musculoskelet Med 4: 23-32.

This article was originally published in a special issue, Hereditary Disorders handled by Editor(s). Dr. X. Long Zheng, University of Pennsylvania, USA 